\title{
Mode mapping in paraxial lossless optics
}

\author{
Tatiana Alieva \\ Facultad de Ciencias Físicas, Universidad Complutense de Madrid, Ciudad Universitaria s/n, Madrid 28040, Spain
}

Martin J. Bastiaans

Faculteit Elektrotechniek, Technische Universiteit Eindhoven, Postbus 513, 5600 MB Eindhoven, The Netherlands

Received December 23, 2004

A Collins transformation maps an orthonormal set of Hermite-Gaussian modes into an orthonormal set of beams with a Gaussian envelope. Among these beams are Laguerre-Gaussian beams and the recently introduced Hermite-Laguerre-Gaussian beams. Compact expressions for the complex field amplitudes of these modes are derived. The results obtained are useful for description of the propagation of light through first-order optical systems, for the solution of the phase-retrieval problem by noninterferometric techniques, and for the design of mode converters and information processing systems. (C) 2005 Optical Society of America

OCIS codes: $070.2580,070.4690,030.4070,120.4820,140.3300$.

Paraxial optical systems are actively used for information processing, for phase retrieval, and for transformation and characterization of optical beams. In particular, the generation of vortex beams attracts much interest of the scientific community because of the possible applications of these beams in microobject manipulation, communication, etc. New families of Gaussian beams ${ }^{1,2}$ that are closely related to Hermite-Gaussian (HG) and Laguerre-Gaussian (LG) beams and new schemes for laser mode converters, such as fractional Fourier-transform (FT) $\pi / 2$ converters, ${ }^{3}$ have recently been proposed. Here we define a general class of Gaussian beams, $A B C D \mathrm{HG}$ modes, obtained from the HG or LG modes after propagation through a first-order optical system described by a Collins integral. ${ }^{4}$

A lossless paraxial system (or first-order optical ABCD system with real matrices ABCD) is described by its symplectic ray transformation matrix. $\mathbf{T},{ }^{5}$ which relates position $\mathbf{r}_{i}=\left(x_{i}, y_{i}\right)^{t}$ and direction $\mathbf{q}_{i}=\left(u_{i}, v_{i}\right)^{t}$ of an incoming ray to position $\mathbf{r}_{o}=\left(x_{o}, y_{o}\right)^{t}$ and direction $\mathbf{q}_{o}=\left(u_{o}, v_{o}\right)^{t}$ of the outgoing ray:

$$
\left(\begin{array}{l}
\mathbf{r}_{o} \\
\mathbf{q}_{o}
\end{array}\right)=\left[\begin{array}{ll}
\mathbf{A} & \mathbf{B} \\
\mathbf{C} & \mathbf{D}
\end{array}\right]\left(\begin{array}{l}
\mathbf{r}_{i} \\
\mathbf{q}_{i}
\end{array}\right)=\mathbf{T}\left(\begin{array}{l}
\mathbf{r}_{i} \\
\mathbf{q}_{i}
\end{array}\right) ;
$$

as usual, the superscript $t$ denotes transposition. The symplecticity of the ABCD matrix yields

$$
\mathbf{A B}^{t}=\mathbf{B} \mathbf{A}^{t}, \quad \mathbf{C D}^{t}=\mathbf{D C}^{t}, \quad \mathbf{A D}^{t}-\mathbf{B} \mathbf{C}^{t}=\mathbf{I} .
$$

Any symplectic matrix is associated with two integral transforms, which differ by $+/-$ some number; see Ref. 6, Sec. 9. Under the assumption that $\mathbf{B}$ is a nonsingular matrix, we can describe the action of a firstorder optical system on complex field amplitude $f_{i}\left(\mathbf{r}_{i}\right)$ at its input by the Collins integral ${ }^{4}$

$$
\begin{aligned}
f_{o}\left(\mathbf{r}_{o}\right)= & \frac{\exp (i \phi)}{\sqrt{\operatorname{det} i \mathbf{B}}} \iint f_{i}\left(\mathbf{r}_{i}\right) \exp \left[i \pi \left(\mathbf{r}_{i}^{t} \mathbf{B}^{-1} \mathbf{A} \mathbf{r}_{i}-2 \mathbf{r}_{i}^{t} \mathbf{B}^{-1} \mathbf{r}_{o}\right.\right. \\
& \left.\left.+\mathbf{r}_{o}^{t} \mathbf{D} \mathbf{B}^{-1} \mathbf{r}_{o}\right)\right] \mathrm{d} \mathbf{r}_{i},
\end{aligned}
$$

where the square root $\sqrt{\operatorname{det} i \mathbf{B}}$ is chosen such that its argument is in the region $[\pi / 2 ; \pi]$. Constant phase factor $\exp (i \phi)$, with which we can cope, if necessary, with the optical path length and the metaplectic sign problem, is rather irrelevant in the scope of this Letter and is dropped in the remainder of it.

There are two sets of orthonormal modes that are widely used in optics: HG and LG modes. An arbitrary square integrable two-dimensional function, representing, for example, complex field amplitude $f(\mathbf{r})$, can be written as a linear superposition of $\mathrm{HG}$ or LG modes. The orthonormal HG mode is given by

$$
\mathcal{H}_{n, m}\left(\mathbf{r} ; w_{x}, w_{y}\right)=\mathcal{H}_{n}\left(x ; w_{x}\right) \mathcal{H}_{m}\left(y ; w_{y}\right),
$$

with

$$
\mathcal{H}_{n}(x ; w)=2^{1 / 4}\left(2^{n} n ! w\right)^{-1 / 2} H_{n}(\sqrt{2 \pi} x / w) \exp \left(-\pi x^{2} / w^{2}\right),
$$

where $H_{n}()$ denotes Hermite polynomials. ${ }^{7}$ The orthonormal form for the LG mode ${ }^{8}$ is

$$
\begin{aligned}
\mathcal{L}_{n, m}(\mathbf{r}, w)= & 2^{1 / 2} w^{-1}[(\min \{n, m\}) ! /(\max \{n, m\}) !]^{1 / 2} \\
& \times(\sqrt{2 \pi} r / w)^{|n-m|} \exp [i(n-m) \varphi] \\
& \times L_{\min \{n, m\}}{ }^{(|n-m|)}\left(2 \pi r^{2} / w^{2}\right) \exp \left[-\pi r^{2} / w^{2}\right],
\end{aligned}
$$

where $x=r \cos \varphi$ and $y=r \sin \varphi$ and where $L_{n}{ }^{(\alpha)}()$ denotes the generalized Laguerre polynomial.

There are several first-order optical systems that are special with respect to the HG and LG modes. One of them is the separable fractional FT system, described by its ray transformation matrix ${ }^{6} \mathbf{T}_{f \mathrm{FT}}$ : 


$$
\left[\begin{array}{cccc}
\cos \gamma_{x} & 0 & w_{x}{ }^{2} \sin \gamma_{x} & 0 \\
0 & \cos \gamma_{y} & 0 & w_{y}{ }^{2} \sin \gamma_{y} \\
-w_{x}{ }^{-2} \sin \gamma_{x} & 0 & \cos \gamma_{x} & 0 \\
0 & -w_{y}{ }^{-2} \sin \gamma_{y} & 0 & \cos \gamma_{y}
\end{array}\right]
$$

for which the HG and LG modes are eigenfunctions of the corresponding Collins transformation. Note that, for the (rotationally symmetric) LG modes, the fractional Fourier transformer should be isotropic: $w_{x}$ $=w_{y}=w$ and $\gamma_{x}=\gamma_{y}=\gamma$.

Other first-order optical systems-mode converters-transform, for instance, HG modes into LG modes and vice versa. To treat systems that convert HG modes $\mathcal{H}_{n, m}\left(\mathbf{r} ; w_{x}, w_{y}\right)$ into other modes, we introduce normalized and dimensionless ray transformation submatrices $\mathbf{a}, \mathbf{b}, \mathbf{c}$, and $\mathbf{d}$ as

$$
\left[\begin{array}{ll}
\mathbf{a} & \mathbf{b} \\
\mathbf{c} & \mathbf{d}
\end{array}\right]=\left[\begin{array}{cc}
\mathbf{W}_{o}^{-1} \mathbf{A} \mathbf{W}_{i} & \mathbf{W}_{o}^{-1} \mathbf{B} \mathbf{W}_{i}^{-1} \\
\mathbf{W}_{o} \mathbf{C} \mathbf{W}_{i} & \mathbf{W}_{o} \mathbf{D} \mathbf{W}_{i}^{-1}
\end{array}\right]
$$

with

$$
\mathbf{W}_{i}=\left[\begin{array}{cc}
w_{x} & 0 \\
0 & w_{y}
\end{array}\right]
$$

where $w_{x}$ and $w_{y}$ are the widths of the HG mode at the system's input, and with $\mathbf{W}_{o}$ a similar diagonal matrix, appropriately chosen, at its output. Several HG-to-LG mode converters ${ }^{1,3,8,9}$ have been proposed; we mention

$$
\begin{gathered}
\mathbf{a}_{1}=\mathbf{d}_{1}=\frac{1}{\sqrt{2}}\left[\begin{array}{ll}
0 & 1 \\
1 & 0
\end{array}\right], \quad \mathbf{b}_{1}=-\mathbf{c}_{1}=\frac{1}{\sqrt{2}}\left[\begin{array}{ll}
1 & 0 \\
0 & 1
\end{array}\right], \\
\mathbf{a}_{2}=\mathbf{d}_{2}=\frac{1}{\sqrt{2}}\left[\begin{array}{ll}
1 & 0 \\
0 & 1
\end{array}\right], \quad \mathbf{b}_{2}=-\mathbf{c}_{2}=\frac{1}{\sqrt{2}}\left[\begin{array}{cc}
0 & -1 \\
-1 & 0
\end{array}\right] .
\end{gathered}
$$

Case 1 is described in Ref. 1, Eq. (6), with $\alpha=\pi / 4$; case 2 is described in Ref. 8, Eq. (14), with $\phi=\pi / 4$. The two systems are related by a Fourier transformation. The LG beams at the output of these systems differ from one another only by a constant phase factor. The inverses of these operations perform a LGto-HG transformation.

Our goal is to find the expression for the beams at the output of an ABCD system starting from the orthonormal HG modes. Note that one could also start from the set of LG modes and obtain the same class of beams, based on the additivity of first-order systems and knowledge of the LG-HG converter matrix. We use the following notation: for a $\mathrm{HG}$ input beam $\mathcal{H}_{n, m}\left(\mathbf{r} ; w_{x}, w_{y}\right)$ [Eqs. (4) and (5)] we get the $A B C D$ $\mathrm{HG}$ beam $\mathcal{H}_{n, m}{ }^{A B C D}\left(\mathbf{r} ; w_{x}, w_{y}\right)$ at the output of an ABCD system after applying Collins integral Eq. (3).

Let us now derive the expression for the complex field amplitude of the beam $\mathcal{H}_{n, m}{ }^{A B C D}\left(\mathbf{r} ; w_{x}, w_{y}\right)$. Applying the Collins integral to the generating function of the HG modes, we can find the generating function of the $A B C D \mathrm{HG}$ beams:

$$
\begin{aligned}
\sum_{n=0}^{\infty} \sum_{m=0}^{\infty} \mathcal{H}_{n, m}{ }^{A B C D}\left(\mathbf{r} ; w_{x}, w_{y}\right)\left(\frac{2^{n+m}}{n ! m !}\right)^{1 / 2} s_{x}{ }^{n} s_{y}{ }^{m} \\
=2^{1 / 2} /\left[\operatorname{det} \mathbf{W}_{o} \operatorname{det}(\mathbf{a}+i \mathbf{b})\right]^{1 / 2} \exp \left[-\mathbf{s}^{t}(\mathbf{a}+i \mathbf{b})^{-1}\right. \\
\left.\quad \times(\mathbf{a}-i \mathbf{b}) \mathbf{s}+2 \sqrt{2 \pi} \mathbf{s}^{t}(\mathbf{a}+i \mathbf{b})^{-1} \boldsymbol{\rho}\right] \\
\quad \times \exp \left[-\pi \boldsymbol{\rho}^{t}(\mathbf{d}-i \mathbf{c})(\mathbf{a}+i \mathbf{b})^{-1} \boldsymbol{\rho}\right],
\end{aligned}
$$

where $\mathbf{s}=\left(s_{x}, s_{y}\right)^{t}$ and where we have introduced normalized and dimensionless space variables $\boldsymbol{\rho}=(\xi, \eta)^{t}$ $=\mathbf{W}_{o}{ }^{-1} \mathbf{r}$. Subsequently we find the derivative and recurrence relations of this generalized class of Gaussian beams, ${ }^{9}$ by differentiating generating function (10) with respect to $\boldsymbol{\rho}$ and $\mathbf{s}$, respectively:

$$
\begin{aligned}
{\left[\frac{\partial}{\partial \xi},\right.} & \left.\frac{\partial}{\partial \eta}\right]^{t} \mathcal{H}_{n, m}{ }^{A B C D}(\mathbf{r})=2 \sqrt{\pi}\left(\mathbf{a}^{t}+i \mathbf{b}^{t}\right)^{-1} \\
\times & {\left[\sqrt{n} \mathcal{H}_{n-1, m}{ }^{A B C D}(\mathbf{r}), \sqrt{m} \mathcal{H}_{n, m-1}{ }^{A B C D}(\mathbf{r})\right]^{t} } \\
& -2 \pi \mathcal{H}_{n, m}{ }^{A B C D}(\mathbf{r})(\mathbf{d}-i \mathbf{c})(\mathbf{a}+i \mathbf{b})^{-1}[\xi, \eta]^{t}, \\
2 \sqrt{\pi} & {[\xi, \eta]^{t} \mathcal{H}_{n, m}{ }^{A B C D}(\mathbf{r})=(\mathbf{a}+i \mathbf{b}) } \\
& \times\left[\sqrt{n+1} \mathcal{H}_{n+1, m}{ }^{A B C D}(\mathbf{r}), \sqrt{m+1} \mathcal{H}_{n, m+1}^{A B C D}(\mathbf{r})\right]^{t} \\
+ & (\mathbf{a}-i \mathbf{b})\left[\sqrt{n} \mathcal{H}_{n-1, m}{ }^{A B C D}(\mathbf{r}), \sqrt{m} \mathcal{H}_{n, m-1}^{A B C D}(\mathbf{r})\right]^{t} .
\end{aligned}
$$

The combination of Eqs. (11) and (12) leads to the following relationship for the $A B C D$ HG beams:

$$
\begin{gathered}
2 \sqrt{\pi(n+1)} \mathcal{H}_{n+1, m}=\mathcal{P} \mathcal{H}_{n, m}, \\
2 \sqrt{\pi(m+1)} \mathcal{H}_{n, m+1}=\mathcal{Q} \mathcal{H}_{n, m},
\end{gathered}
$$

with the operators

$$
\begin{aligned}
& \mathcal{P}=2 \pi\left(U_{11} \xi+U_{12} \eta\right)-Z_{11} \frac{\partial}{\partial \xi}-Z_{12} \frac{\partial}{\partial \eta} \\
& \mathcal{Q}=2 \pi\left(U_{21} \xi+U_{22} \eta\right)-Z_{21} \frac{\partial}{\partial \xi}-Z_{22} \frac{\partial}{\partial \eta}
\end{aligned}
$$

and the matrices

$$
\begin{aligned}
& \mathbf{U}=(\mathbf{a}-i \mathbf{b})^{t}\left[(\mathbf{d}-i \mathbf{c})(\mathbf{a}+i \mathbf{b})^{-1}\right]^{*}, \\
& \mathbf{Z}=(\mathbf{a}-i \mathbf{b})^{t}
\end{aligned}
$$

Note that the operators $\mathcal{P}$ and $\mathcal{Q}$ commute because $\mathbf{Z} \mathbf{U}^{t}=\mathbf{U} \mathbf{Z}^{t}$ and that we are thus led to an alternative definition of the $A B C D \mathrm{HG}$ beams:

$$
\mathcal{H}_{n, m}{ }^{A B C D}(\mathbf{r})=\frac{\mathcal{P}^{n} \mathcal{Q}^{m} \mathcal{H}_{0,0}{ }^{A B C D}(\mathbf{r})}{2^{n+m}\left(\pi^{n+m} n ! m !\right)^{1 / 2}} .
$$

Based on the formula ${ }^{9} \iint \exp \left(-\pi \mathbf{r}_{i}^{t} \mathbf{P} \mathbf{r}_{i}-i 2 \pi \mathbf{r}_{i}^{t} \mathbf{q}\right) \mathrm{d} \mathbf{r}_{i}$ $=\exp \left(-\pi \mathbf{q}^{t} \mathbf{P}^{-1} \mathbf{q}\right) / \sqrt{\operatorname{det} \mathbf{P}}$ for the calculation of the Collins integral for the $\mathrm{HG}$ fundamental mode $\mathcal{H}_{0,0}\left(\mathbf{r} ; w_{x}, w_{y}\right)$, an explicit form for the fundamental ( $n=m=0) A B C D$ HG mode is found: 


$$
\begin{aligned}
\mathcal{H}_{0,0}{ }^{A B C D}(\mathbf{r})= & 2^{1 / 2} /\left[\operatorname{det} W_{o} \operatorname{det}(\mathbf{a}+i \mathbf{b})\right]^{1 / 2} \\
& \times \exp \left[-\pi \boldsymbol{\rho}^{t}(\mathbf{d}-i \mathbf{c})(\mathbf{a}+i \mathbf{b})^{-1} \boldsymbol{\rho}\right] .
\end{aligned}
$$

One can easily confirm the orthonormality relationship between $\mathcal{H}_{n, m}{ }^{A B C D}\left(\mathbf{r} ; w_{x}, w_{y}\right)$ and $\mathcal{H}_{l, k}{ }^{A B C D}\left(\mathbf{r} ; w_{x}, w_{y}\right)$ :

$$
\begin{aligned}
\iint & \mathcal{H}_{n, m}{ }^{A B C D}\left(\mathbf{r} ; w_{x}, w_{y}\right)\left[\mathcal{H}_{l, k}{ }^{A B C D}\left(\mathbf{r} ; w_{x}, w_{y}\right)\right]^{*} \mathrm{~d} \mathbf{r} \\
= & \delta_{n l} \delta_{m k}
\end{aligned}
$$

which is a direct consequence of the orthonormality relationship between the HG beams $\mathcal{H}_{n, m}\left(\mathbf{r} ; w_{x}, w_{y}\right)$ and $\mathcal{H}_{l, k}\left(\mathbf{r} ; w_{x}, w_{y}\right)$ at the input of the first-order optical system and the unitarity of this system.

Note that if ray transformation matrix $\mathbf{T}$ commutes with the ray transformation matrix $\mathbf{T}_{\mathrm{FT}}$ of a FT system ${ }^{6}$ [cf. relation (7) with $\gamma_{x}=\gamma_{y}=\pi / 2$ and $w_{x}$ $\left.=w_{y}\right]$ we have $\mathbf{d}-i \mathbf{c}=\mathbf{a}+i \mathbf{b}$ and therefore $\mathbf{U}=\mathbf{Z}$. In this case the operators reduce to

$$
\begin{aligned}
& \mathcal{P}=-Z_{11}(\partial / \partial \xi-2 \pi \xi)-Z_{12}(\partial / \partial \eta-2 \pi \eta), \\
& \mathcal{Q}=-Z_{21}(\partial / \partial \xi-2 \pi \xi)-Z_{22}(\partial / \partial \eta-2 \pi \eta),
\end{aligned}
$$

and the $A B C D$ HG beams reduce to the twodimensional HG modes considered in Ref. 2. Inasmuch as

$$
\left(\frac{\mathrm{d}}{\mathrm{d} t}-2 \pi t\right)^{n} \exp \left(-\pi t^{2}\right)=\exp \left(\pi t^{2}\right)\left(\frac{\mathrm{d}}{\mathrm{d} t}\right)^{n} \exp \left(-2 \pi t^{2}\right)
$$

the beams then may as well be expressed in the form

$$
\begin{aligned}
\mathcal{H}_{n, m}{ }^{A B C D}(\mathbf{r})= & \frac{2^{1 / 2}(-1)^{n+m} \exp \left[\pi\left(\xi^{2}+\eta^{2}\right)\right]}{2^{n+m}\left[\pi^{n+m} n ! m ! \operatorname{det} \mathbf{W}_{o} \operatorname{det}(\mathbf{a}+i \mathbf{b})\right]^{1 / 2}} \\
& \times\left(Z_{11} \frac{\partial}{\partial \xi}+Z_{12} \frac{\partial}{\partial \eta}\right)^{n}\left(Z_{21} \frac{\partial}{\partial \xi}+Z_{22} \frac{\partial}{\partial \eta}\right)^{m} \\
& \times \exp \left[-2 \pi\left(\xi^{2}+\eta^{2}\right)\right] .
\end{aligned}
$$

The recently introduced Hermite-LaguerreGaussian modes ${ }^{1}$ are particular examples of twodimensional HG modes and are therefore $A B C D$ HG modes. They can be obtained from HG modes by the Collins transformation parameterized by the matrices

$$
\begin{aligned}
& \mathbf{a}=\mathbf{d}=\frac{1}{\sqrt{2}}\left[\begin{array}{cc}
\cos 2 \alpha & \sin 2 \alpha \\
\sin 2 \alpha & -\cos 2 \alpha
\end{array}\right], \\
& \mathbf{b}=-\mathbf{c}=\frac{1}{\sqrt{2}}\left[\begin{array}{ll}
1 & 0 \\
0 & 1
\end{array}\right] .
\end{aligned}
$$

Simple expressions of these modes, which for $\alpha$ $=\pi / 4$ reduce to LG modes, can be obtained from Eq. (21). [Note that in Ref. 1 the output field amplitude
$\mathcal{G}_{n, m}(\mathbf{r} \mid \alpha)$ is defined with an additional rotation of the coordinate system through angle $\alpha$.]

Because HG modes are eigenfunctions of separable fractional FT (7) with unimodular eigenvalues, any system described by the matrix $\mathbf{T}_{A B C D} \mathbf{T}_{f \mathrm{FT}}$ with $\mathcal{H}_{n, m}\left(\mathbf{r} ; w_{x}, w_{y}\right)$ at its input produces at its output the beam $\mathcal{H}_{n, m}{ }^{A B C D}\left(\mathbf{r} ; w_{x}, w_{y}\right)$ with an additional constant phase shift. This fact enlarges the number of optical configurations used to produce certain kinds of beams, for example, HG-to-LG converters. Moreover, as the two systems characterized by $\mathbf{T}_{A B C D}$ and $\mathbf{T}_{A B C D} \mathbf{T}_{f \mathrm{FT}}$ produce - up to the constant phase shiftthe same output $\mathcal{H}_{n, m}{ }^{A B C D}\left(\mathbf{r} ; w_{x}, w_{y}\right)$ from a HG mode at their inputs, this implies that the $A B C D$ HG mode is an eigenfunction of the Collins transform corresponding to the matrix $\mathbf{T}=\mathbf{T}_{A B C D} \mathbf{T}_{f \mathrm{FT}} \mathbf{T}_{A B C D}{ }^{-1}$.

We can also use the orthonormality of the $A B C D$ HG modes to describe the evolution of an arbitrary complex field during its propagation through a firstorder optical system. Because the HG modes form a complete set, any function $f_{i}(\mathbf{r})$ can be decomposed as

$$
\begin{aligned}
f_{i}(\mathbf{r}) & =\sum_{m} \sum_{n} q_{n m} \mathcal{H}_{n, m}\left(\mathbf{r} ; w_{x}, w_{y}\right), \\
q_{n m} & =\iint\left[\mathcal{H}_{n, m}\left(\mathbf{r} ; w_{x}, w_{y}\right)\right]^{*} f_{i}(\mathbf{r}) \mathrm{d} \mathbf{r} .
\end{aligned}
$$

Every $\mathrm{HG}$ mode $\mathcal{H}_{n, m}\left(\mathbf{r} ; w_{x}, w_{y}\right)$ maps into the $\mathcal{H}_{n, m}{ }^{A B C D}\left(\mathbf{r} ; w_{x}, w_{y}\right)$ mode at the output of the ABCD system. Then we can write the expression for the complex field amplitude in the output plane in the form

$$
f_{o}(\mathbf{r})=\sum_{m} \sum_{n} q_{n m} \mathcal{H}_{n, m}{ }^{A B C D}\left(\mathbf{r} ; w_{x}, w_{y}\right) .
$$

This is a generalization of the expression used for the propagation through a fractional FT system to the case of a general ABCD system. The mapping of orthonormal modes can be used for phase recovery from intensity distribution, for analysis of imaging systems, and for beam design.

T. Alieva (talieva@fis.ucm.es) thanks the Spanish Ministry of Education and Science for financial support (Ramon y Cajal grant and projects TIC 200201846 and TIC 2002-11581-E). M. J. Bastiaans's e-mail address is m.j.bastiaans@tue.nl.

\section{References}

1. E. G. Abramochkin and V. G. Volostnikov, J. Opt. A, Pure Appl. Opt. 6, S157 (2004).

2. A. Wünsche, J. Phys. A 33, 1603 (2000).

3. A. A. Malyutin, Quantum Electron. 34, 165 (2004).

4. S. A. Collins, Jr., J. Opt. Soc. Am. 60, 1168 (1970).

5. R. K. Luneburg, Mathematical Theory of Optics (U. California Press, 1966).

6. R. Simon and K. B. Wolf, J. Opt. Soc. Am. A 17, 2368 (2000).

7. M. Abramowitz and I. A. Stegun eds., Pocketbook of Mathematical Functions (Harri Deutsch, 1984).

8. R. Simon and G. S. Agarwal, Opt. Lett. 25, 1313 (2000).

9. M. J. Bastiaans and T. Alieva, J. Phys. A 38, L73 (2005). 